\title{
Neutrophil-to-lymphocyte ratio, blood eosinophils and COPD exacerbations: a cohort study
}

\author{
Jens Ellingsen $\mathbb{Q}^{1}$, Christer Janson $\mathbb{C}^{1}$, Kristina Bröms $\mathbb{B}^{2}$, Karin Lisspers ${ }^{2}$, Björn Ställberg ${ }^{2}$, \\ Marieann Högman ${ }^{1}$ and Andrei Malinovschi (i) ${ }^{3}$
}

${ }^{1}$ Dept of Medical Sciences, Respiratory, Allergy and Sleep Research, Uppsala University, Uppsala, Sweden. ${ }^{2}$ Dept of Public Health and Caring Sciences, Family Medicine and Preventive Medicine, Uppsala University, Uppsala, Sweden. ${ }^{3}$ Dept of Medical Sciences, Clinical Physiology, Uppsala University, Uppsala, Sweden.

Corresponding author: Jens Ellingsen (jens.ellingsen@medsci.uu.se)

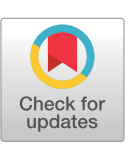

This version is distributed under the terms of the Creative Commons Attribution Non-Commercial Licence 4.0. For commercial reproduction rights and permissions contact permissions@ersnet.org

This article has supplementary material available from openres.ersjournals.com

Received: 19 July 2021 Accepted: 24 Oct 2021

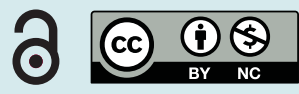

Shareable abstract (@ERSpublications)

Neutrophil-to-lymphocyte ratio and blood eosinophils in stable-phase COPD are associated with future exacerbations and show fair longitudinal reliability https://bit.ly/3CAS1jY

Cite this article as: Ellingsen J, Janson C, Bröms K, et al. Neutrophil-to-lymphocyte ratio, blood eosinophils and COPD exacerbations: a cohort study. ERJ Open Res 2021; 7: 00471-2021 [DOI: 10.1183/ 23120541.00471-2021].

\section{Abstract}

Background Blood neutrophil-to-lymphocyte ratio (NLR) and blood eosinophils (B-Eos) are emerging biomarkers in COPD. This study examined whether they could predict acute exacerbations of COPD (AECOPDs), and determined their longitudinal stability.

Methods In this closed cohort study, Swedish subjects with spirometry-verified COPD attended three yearly visits in a stable phase of the disease. Blood cell counts, spirometry and questionnaire-assessed AECOPD-history (worsening of COPD leading to an unscheduled visit and/or use of antibiotics and/or oral corticosteroids) were collected at each visit.

Results Of 466 included subjects $57 \%$ were female. Baseline mean \pm sD forced expiratory volume in $1 \mathrm{~s}$ was $58 \pm 17 \%$ predicted. High NLR $(\geqslant 3.0)$ was more common in subjects with previous AECOPDs than in those without $(33.5 \%$ versus $20.4 \%, \mathrm{p}=0.002)$. In two-level mixed-effects logistic regression models adjusted for confounders, NLR as a continuous variable (OR 1.20, 95\% CI 1.04-1.38) and B-Eos $\geqslant 300$ cells $\cdot \mu \mathrm{L}^{-1}$ (OR 1.54, 95\% CI 1.06-2.24) were associated with future AECOPDs. In 386 subjects with blood cell data available at all three visits, the intraclass correlation coefficient for NLR was 0.61 (95\% CI 0.56-0.66) and for B-Eos 0.69 (95\% CI 0.64-0.73). NLR was persistently $\geqslant 3.0$ in $10.6 \%$ and B-Eos was persistently $\geqslant 300$ cells $\cdot \mu \mathrm{L}^{-1}$ in $15.3 \%$.

Conclusions Stable phase NLR and B-Eos were associated with future AECOPDs. NLR on its own is probably not useful to predict AECOPDs but might be included in a risk scoring index. A minority of subjects with COPD had persistently elevated stable-phase NLR or B-Eos, and the biomarkers showed fair longitudinal reliability.

Introduction

COPD is a major cause of morbidity and mortality globally [1]. Acute exacerbations of COPD (AECOPDs) are defined as "acute worsening of respiratory symptoms that result in additional therapy" and contribute substantially to the burden of the disease [2]. AECOPDs are associated with an accelerated decrease in lung function, lowered quality of life, increased mortality and increased healthcare costs [3-5]. Preventing and treating AECOPDs is a major goal in the management of COPD patients.

Blood eosinophils (B-Eos) in COPD have attracted great scientific interest in recent years. Observational studies associate elevated B-Eos with an increased number of AECOPDs [6], though results are contradictory [7, 8]. Several post hoc analyses from clinical trials indicate that higher levels of B-Eos might predict response to treatment with inhaled corticosteroids (ICS) [9, 10]. These and other results [11] have led to the incorporation of B-Eos into international guidelines as a biomarker to guide pharmacological treatment decisions, i.e. the prescription of ICS [2]. However, the role of B-Eos as a 
clinically useful biomarker is under debate [12], and there are results contradicting current guidelines [13]. Moreover, antibody-drugs targeting interleukin-5 or its receptor and thereby eosinophilic inflammation, successfully used in severe eosinophilic asthma, have modest or no effects in COPD [14, 15].

In recent years, the relationship between blood neutrophils (B-Neu) and blood lymphocytes (B-Lym), i.e. the neutrophil-to-lymphocyte ratio (NLR), has emerged as a potential biomarker in COPD. An elevated NLR in stable-phase COPD compared to healthy controls was reported for the first time in 2013 [16], and in AECOPD compared to stable-phase COPD 2014 [17]. These findings have been repeated in several studies and confirmed in a meta-analysis [18]. A systematic review found that NLR is associated with a diagnosis of AECOPD and a predictor of mortality [19]. Another meta-analysis suggests that NLR can predict future AECOPDs and mortality in COPD [20]. However, all these reviews and meta-analyses conclude that data are scarce and that larger studies are needed to evaluate the clinical usefulness of NLR.

An important issue when introducing biomarkers in treatment algorithms is longitudinal stability. As for B-Eos, the proportion of subjects with persistently high levels varies from $5 \%$ to $45 \%$ in previous works [8, 21-25]. The longitudinal reliability of B-Eos is reported to be good in three previous studies [25-27]. Only one previous study in subjects with COPD has examined the longitudinal stability of NLR [28]. However, that study did not examine the longitudinal reliability.

The aims of this study were: 1) to test the hypothesis that NLR and/or B-Eos can predict future AECOPD; and 2) to determine the longitudinal stability and reliability of NLR and B-Eos.

Methods

Ethical approval

The study was approved by the Regional Ethics Review Board in Uppsala, Sweden (Dnr 2013/358). Written informed consent was obtained from all participants.

\section{Study design, setting and participants}

The Tools Identifying Exacerbations in COPD (TIE) study was a closed cohort study conducted at three study sites in different Swedish regions (Dalarna, Gävleborg and Uppsala) with the overall aim to investigate predictors of AECOPDs. As previously described [29], subjects with spirometry-verified COPD were recruited from primary and secondary care between September 2014 and September 2016. They attended annual stable-phase (at least 4 weeks since the last AECOPD) follow-up visits for 2 years (i.e. three visits: baseline, year 1, and year 2) with completion of questionnaires, pulmonary function testing and blood samples. Inclusion and exclusion criteria are shown in figure 1. Follow-up was completed in October 2018. Study period for the present analyses is defined as the three-year period from 12 months before inclusion to the 2-year follow-up visit.

For the analyses of the ability of blood cells to predict AECOPD (AECOPD analyses), subjects with complete data on the following variables at one or more visits were included: AECOPD during the following year, history of AECOPD the past year, B-Eos, NLR, COPD Assessment Test (CAT) [30] score, body mass index (BMI), current smoking, current use of ICS, forced expiratory volume in $1 \mathrm{~s}\left(\mathrm{FEV}_{1}\right)$, sex and age. For the analyses of longitudinal blood cell stability and reliability (stability analyses), only those with complete data on blood cells at all three visits were included.

\section{Variables}

The participants completed the CAT and a questionnaire on demographics, current smoking status, comorbidities, regular COPD medication, healthcare contacts including acute visits, and any courses of oral corticosteroids (OCS) or antibiotics for AECOPD. Current smoking was defined as ongoing regular or sporadic smoking at the time of visit. Self-reported comorbidities, current or previous, were assessed at baseline visit only and included asthma, chronic bronchitis, coronary heart disease, heart failure, and depression or anxiety. Current medication use was defined as regular use in the last 6 months, alone or in combination with other drugs. BMI was calculated as the ratio of weight $(\mathrm{kg})$ to height $(\mathrm{m})$ squared. Spirometry was performed $15 \mathrm{~min}$ after inhalation of $400 \mu \mathrm{g}$ salbutamol as previously described [29]. Swedish reference values were used to express $\mathrm{FEV}_{1}$ as per cent predicted [31, 32]. Blood cell counts including total blood leukocytes, B-Neu, B-Lym, B-Eos and thrombocytes were analysed, as previously described [29]. NLR was calculated as the ratio of B-Neu to B-Lym. Because of lack of established threshold values, the upper quartile of NLR at baseline was arbitrarily used to define "high NLR" as 23.00. B-Eos values were categorised as low, intermediate or high $(<150, \geqslant 150-<300$ and $\geqslant 300$ cells $\cdot \mu \mathrm{L}^{-1}$, respectively) (supplementary appendix A1 and figure S1). For some analyses, dichotomised B-Eos categories were used $\left(<300\right.$ and $\geqslant 300$ cells $\left.\cdot \mu \mathrm{L}^{-1}\right)$. 


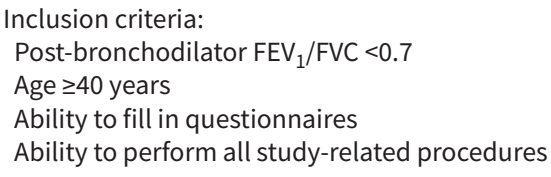

Exclusion criteria:

Severe comorbidity as assessed by study physican Inability to participate in all parts of the study

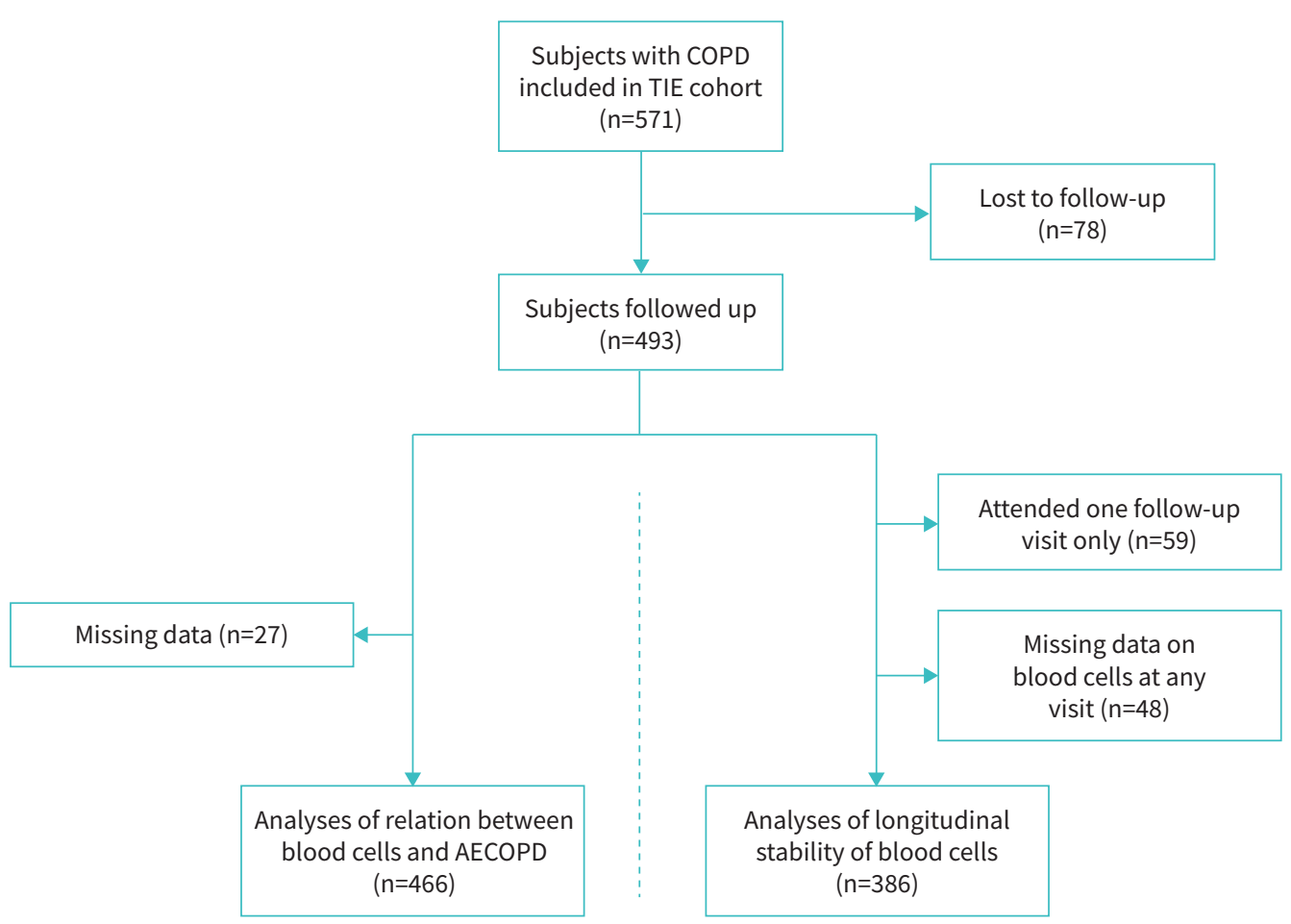

FIGURE 1 Flow diagram of the inclusion process. FEV ${ }_{1}$ : forced expiratory volume in $1 \mathrm{~s}$; FVC: forced vital capacity; TIE: Tools Identifying Exacerbations in COPD study; AECOPD: acute exacerbation of COPD.

AECOPD was the outcome of this study and was assessed through questionnaires covering the previous 1-year period. AECOPD was defined as an acute healthcare visit and/or prescription of OCS and/or antibiotics due to worsening of COPD.

\section{Statistical analysis}

Subjects lost to follow-up were excluded from analysis, whereas those with incomplete follow-up (visiting only one of the follow-ups) were included in the AECOPD analyses but not in the stability analyses. Assuming data were missing completely at random, subjects with missing data were excluded since they were relatively few (supplementary tables S3 and S4).

Data were described using number (n) and percentage, mean \pm SD or median and interquartile range (IQR). Comparisons between groups were made with Pearson's chi-squared test. The significance level was set to 0.05 .

Correlation between variables was assessed at baseline using Pearson correlation coefficient or Spearman's rank correlation coefficient (rho), as appropriate. Two-level (repeated measures in the same individual) mixed-effects logistic regression models with future AECOPD within a year as the outcome and blood cells as predictors were constructed to produce odds ratios with 95\% confidence intervals. The models were then adjusted by including AECOPD previous year, CAT score, BMI, current smoking, current ICS use, $\mathrm{FEV}_{1}$, sex and age as predictors. The adjusting predictors were chosen since they are known risk factors for AECOPD and are therefore likely to confound the estimations. The calculations were repeated in a three-level model by adding study-site as a cluster-level, which yielded nearly identical results. Therefore, we deemed that data were independent of the study-site, and accordingly that there was no need to cluster subjects. Therefore we present the two-level models throughout the paper. To analyse subgroups, various stratifications were performed. The presence of interactions was analysed by fitting models with 
interaction terms of the blood cell variable of interest by the other variables, one-by-one. The interaction-models were compared to the main model using the likelihood-ratio test. In a sensitivity analysis, the inclusion criteria were adjusted by omitting current smokers from the analysis.

Owing to non-normality (supplementary figure S1), blood cell data were log-transformed for the analyses of longitudinal reliability. Because $\geqslant 1$ B-Eos value was zero in 15 subjects, log-transformed B-Eos data were missing for these subjects (i.e. $\mathrm{n}=371$ instead of 386). Two-way mixed-effects model single-measurement absolute-agreement intraclass correlation coefficients (ICC) with 95\% confidence intervals were calculated for the reliability of repeated measurements in the same subject [33]. A proposed interpretation of ICC values is: excellent $(>0.75)$, fair to good $(0.40-0.75)$ or poor $(<0.40)$ [26].

Data management and statistical analyses were performed using Stata Statistical Software: Release 14 (StataCorp LP 2015, College Station, TX, USA).

\section{Results}

Participants

Of the 571 subjects who were included in the TIE cohort, 493 were followed up at least once (figure 1). In the AECOPD analyses, 466 subjects were included, and in the stability analyses 386.

\section{Descriptive data}

In the AECOPD analyses, 57.5\% were female, and 26.2\% were currently smoking (table 1). Among subjects with high NLR, a history of AECOPD was more common, CAT was higher and high B-Eos was less common than among subjects with low NLR. Baseline-characteristics of the population in the stability analyses are presented in supplementary tables S1 and S2.

Stratification of baseline blood cell levels by AECOPD history (figure 2) showed that high NLR was more common in subjects with previous AECOPDs than in those without ( $\mathrm{p}=0.002)$, whereas no such difference existed regarding $\mathrm{B}-\mathrm{Eos}(\mathrm{p}=0.88)$.

Subjects not included in the analyses were of similar age, but more often female and current smokers than included subjects (supplementary tables S3 and S4). The proportion of subjects with a history of AECOPD was larger, $\mathrm{FEV}_{1}$ and BMI were lower, and CAT was higher among the non-included. Moreover, fewer had high B-Eos levels, but high NLR was more common.

\section{Analyses of the relationship between blood cells and AECOPDs}

The AECOPD analyses comprised 881 person-years of follow-up, with a mean follow-up time of 1.9 years. During the 2-year follow-up 206 of 466 subjects (44.2\%) experienced $\geqslant 1$ AECOPD (first year 32.6\%; second year 29.0\%). Rates did not differ between baseline blood cell groups: high NLR 50.4\% versus low 42.0\%, $\mathrm{p}=0.11$; high $\mathrm{B}-$ Eos $50.4 \%$ versus intermediate or low $41.8 \%, \mathrm{p}=0.096$.

At baseline, there were weak correlations between NLR and future AECOPD (Spearman's rho 0.16, $\mathrm{p}<0.001$ ) and NLR and B-Eos (rho -0.12, $\mathrm{p}=0.009$ ), whereas B-Eos and future AECOPD were not correlated (rho $-0.04, \mathrm{p}=0.37$ ). There was no multicollinearity between any of the variables included in the analyses.

Stable-phase NLR as a continuous variable, but not dichotomised, was associated with future AECOPDs in the unadjusted AECOPD analyses (table 2). However, that association was significant only among subjects with $\geqslant 1$ AECOPD the previous year. There was no association between stable-phase B-Eos and future AECOPDs, regardless of AECOPD history (table 2).

In the adjusted AECOPD analyses (table 3), NLR as a continuous variable, but not dichotomised, was associated with future AECOPDs, whereas B-Eos as a dichotomised variable, but not continuous, related to future AECOPDs. Adjusted models stratified by AECOPD history, sex, ICS use, OCS use, and current or previous comorbidity (asthma, chronic bronchitis, coronary heart disease, heart failure, and depression or anxiety) reported at baseline, respectively, are shown in supplementary table S5. Continuous NLR was associated with AECOPD in men but not in women and in subjects without chronic bronchitis but not those with; the opposite was true for dichotomous B-Eos.

No significant interactions were found between stable-phase NLR or B-Eos and the other included variables. In sensitivity analyses where current smokers were excluded from the adjusted models, estimates were similar (data not shown). 
TABLE 1 Characteristics of subjects included in analyses of the association between blood cells and acute exacerbations of COPD, categorised by baseline blood cells

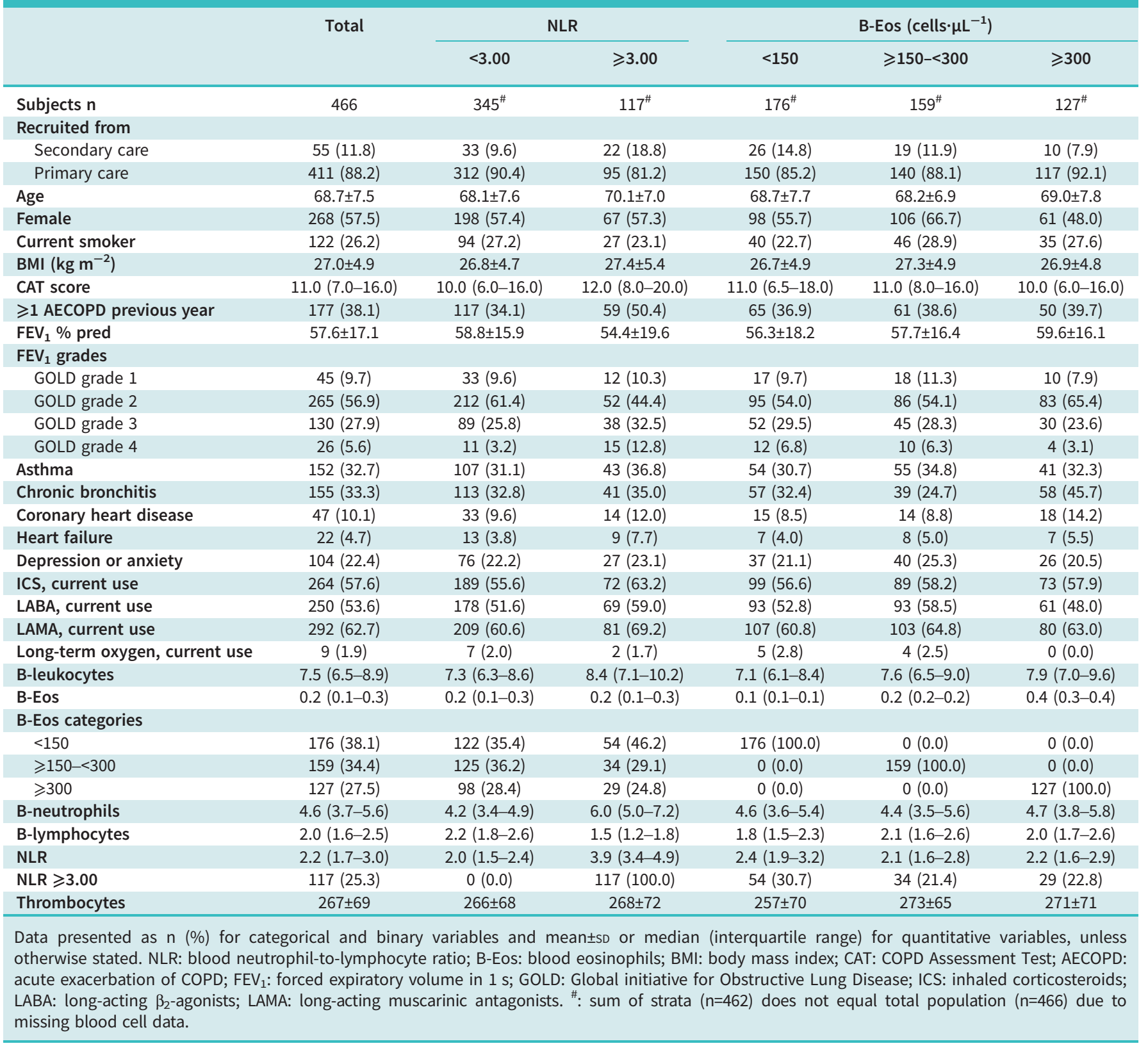

Longitudinal analysis of blood cell stability

The ICC for stable-phase NLR was 0.61 (95\% CI 0.56-0.66). The distribution of NLR at each visit is shown in supplementary table S6. During the study period, 38.9\% had at least one high NLR measurement. Figure 3 shows that $10.6 \%$ had persistently high and $61.1 \%$ persistently low NLR, whereas the remaining $28.3 \%$ changed groups between visits.

The ICC for stable-phase B-Eos was 0.69 (95\% CI 0.64-0.73). The distribution of B-Eos at each visit is shown in supplementary table S6. During the study period, $42.0 \%$ had at least one high B-Eos measurement. Figure 4 shows that $15.3 \%$ had persistently high B-Eos, $10.9 \%$ had persistently intermediate B-Eos and $22.0 \%$ had persistently low B-Eos, whereas the remaining 51.8\% changed groups between visits.

Stratification by any AECOPD during the study period (figure 5) revealed no differences regarding the proportions with persistent levels of blood cells. Stratification by any use of OCS during the study period 

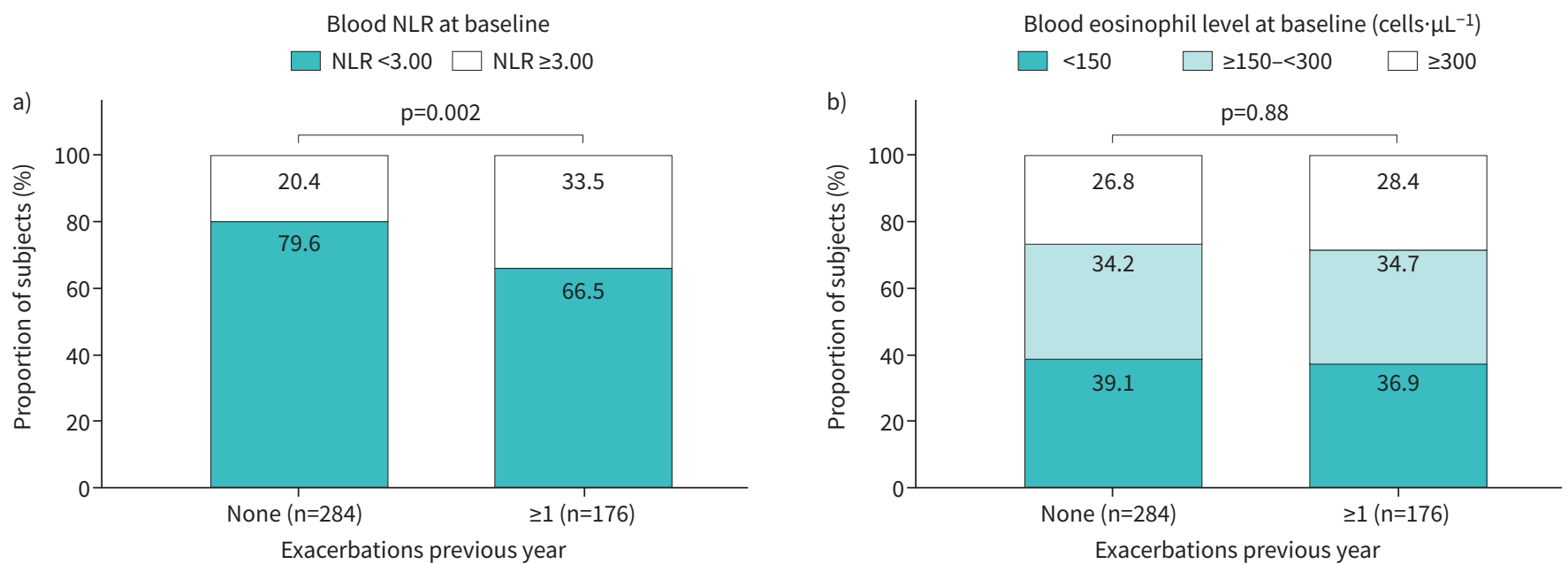

FIGURE 2 Baseline a) blood neutrophil-to-lymphocyte ratio (NLR) and b) blood eosinophils in 460 subjects with COPD, stratified by the occurrence of any exacerbation the previous year.

showed higher proportions of subjects with high and variable NLR among subjects with OCS than those without (data not shown, $\mathrm{p}=0.003)$. For $\mathrm{B}$-Eos, there was no such difference $(\mathrm{p}=0.53)$. There were no significant differences when stratifying by current smoking and current or previous asthma diagnosis at baseline, respectively (data not shown).

\section{Discussion}

Our main findings were that higher stable-phase NLR, analysed as a continuous variable, had an independent prognostic value for future AECOPD, and that stable-phase B-Eos $\geqslant 300$ cells $\mu \mathrm{L}^{-1}$ was associated with future AECOPD. The longitudinal reliability was fairly good with ICCs of 0.61 for NLR and 0.69 for B-Eos. Persistently high NLR and B-Eos at three yearly visits were found in $10.6 \%$ and $15.3 \%$ of the subjects, respectively.

TABLE 2 Mixed-effects single variable logistic regression models on the association of

neutrophil-to-lymphocyte ratio and blood eosinophils to risk of acute exacerbation of COPD the following year

OR $(95 \% \mathrm{Cl})$

\begin{tabular}{|c|c|}
\hline \multicolumn{2}{|l|}{ NLR, continuous variable } \\
\hline Entire study population & $1.48(1.21-1.81)$ \\
\hline No AECOPD previous year & $1.23(0.88-1.72)$ \\
\hline$\geqslant 1$ AECOPD previous year & $1.64(1.14-2.36)$ \\
\hline \multicolumn{2}{|l|}{$\mathrm{NLR} \geqslant 3.00$ versus $<3.00$} \\
\hline Entire study population & $1.61(0.91-2.84)$ \\
\hline No AECOPD previous year & $0.89(0.36-2.22)$ \\
\hline$\geqslant 1$ AECOPD previous year & $2.88(1.00-8.25)$ \\
\hline \multicolumn{2}{|l|}{ B-Eos, continuous variable } \\
\hline Entire study population & $1.02(0.86-1.19)$ \\
\hline No AECOPD previous year & $1.10(0.87-1.37)$ \\
\hline$\geqslant 1$ AECOPD previous year & $0.96(0.72-1.28)$ \\
\hline \multicolumn{2}{|c|}{ B-Eos $\geqslant 300$ versus $<300$ cells $\cdot \mu \mathrm{L}^{-1}$} \\
\hline Entire study population & $1.47(0.83-2.63)$ \\
\hline No AECOPD previous year & $2.10(0.84-5.15)$ \\
\hline$\geqslant 1$ AECOPD previous year & $1.15(0.40-3.28)$ \\
\hline $\begin{array}{l}\text { Models were calculated for } \\
\text { had not had an acute exace } \\
\text { NLR: blood neutrophil-to-lym } \\
\text { \#: per } 100 \text { cells } \mu L^{-1} \text { increase. }\end{array}$ & $\begin{array}{l}\text { ubjects had and } \\
\text { separate model. } \\
\text { ood eosinophils. }\end{array}$ \\
\hline
\end{tabular}


TABLE 3 Mixed-effects multivariable logistic regression models on the association of neutrophil-to-lymphocyte ratio and blood eosinophils with the risk of acute exacerbation of COPD the following year

Models with NLR only aOR $(95 \% \mathrm{Cl})$

\section{NLR, continuous variable}

$\mathrm{NLR} \geqslant 3.00$ versus $<3.00$

B-Eos, continuous variable ${ }^{+}$

B-Eos $\geqslant 300$ versus $<300$
$1.20(1.04-1.38)$

$1.13(0.76-1.68)$

$x$

$x$
Models with B-Eos only aOR $(95 \% \mathrm{Cl})$

$x$

$1.08(0.98-1.20)$

$1.54(1.06-2.24)$
Models combining NLR and B-Eos aOR $(95 \% \mathrm{Cl})$

$1.22(1.06-1.40)$

$1.13(0.76-1.67)$

$1.10(0.997-1.22)$

$1.54(1.06-2.24)$

$x$ indicates predictor not analysed. Models adjusted for $\geqslant 1$ acute exacerbation of COPD preceding year, COPD Assessment Test score, body mass index, current smoking, current inhaled corticosteroid use, forced expiratory volume in $1 \mathrm{~s}$, sex and age. Complete estimates for all predictors are shown in supplementary table S7. NLR: blood neutrophil-to-lymphocyte ratio; B-Eos: blood eosinophils; aOR: adjusted odds ratio. \#: each aOR represents a separate model; ": two models, one for the two continuous variables and one for the two dichotomous; ${ }^{+}$: per 100 cells· $\mu \mathrm{L}^{-1}$ increase

High NLR was related to future AECOPDs in our material. Relatively few previous studies have analysed this. LEE et al. [34] sampled NLR in a Korean cohort of 885 COPD patients (91.4\% men) in a stable phase and found that NLR in the highest quartile (>2.94) was an independent predictor of AECOPDs after 1 year of follow-up. Another Korean cohort comprising 120 COPD patients (92.5\% men) and 28 sex and smoking-history matched healthy controls showed that an NLR in the stable/convalescent phase of $\geqslant 2.8$ (determined by receiver operating characteristic (ROC) analysis) predicts AECOPD hospitalisation. However, this was not statistically significant [35]. SAKURAI et al. [28] followed a Japanese cohort of 274 COPD patients (83\% men) and found that baseline stable-phase NLR $\geqslant 2.7$ (determined by ROC analysis) predicts moderate/severe AECOPD over 3 years.

In the present study, we found that NLR sampled in stable-phase COPD was, as a continuous variable, associated with future self-reported AECOPD during 1 year of follow-up with an OR of 1.20 after adjustment for potential confounders. Our study is not the first to examine this association. Still, it brings important new information as it is the first to include a substantial number of women: the three previous studies together included 132 women [28, 34, 35], whereas this study alone included 268. Our study also contributes a broader geographic distribution; while previous studies were performed in Asia [28, 34, 35], ours was performed in northern Europe. The magnitude of the association between NLR and future AECOPD in this study is substantially lower than that in previous works [28, 34, 35]. A higher proportion of women (57.5\%) can explain part of that difference, as the sex-stratified OR was higher in men. It is also possible that ethnicity explains the disparities between our Swedish cohort and the three Asian ones, since ethnical differences in the ability of NLR to predict mortality in COPD patients [20] and in normal NLR values [36] have been described. The effect of ethnicity on the relation between NLR and AECOPD, however, has not been studied. In our study, the arbitrarily chosen "high" cut-off at $\geqslant 3.00$ did not prove useful in predicting AECOPD, probably because it lacked a clinical rationale. Regardless, it is clear that NLR is associated with future AECOPD, although more work is needed to establish its usefulness and relevant clinical cut-offs. We believe that it is more likely that NLR could be part of a future composite risk scoring system rather than be used on its own to predict AECOPD.

Several studies have found B-Eos to be predictive of AECOPD [6, 9], and a predictive factor for ICS treatment in preventing AECOPD [9]. However, other studies found no relationship between B-Eos and AECOPD [8, 12, 37, 38], and questions its usefulness to guide treatment decisions [12, 13]. We found an association between stable-phase B-Eos and AECOPD only after dichotomisation of B-Eos. That can probably be explained by nonlinear risk increase [9] and a relatively small cohort, compared to previous post hoc analyses of large trials $[9,10]$.

The combination of NLR and B-Eos, sampled in a stable phase, did not increase the ability to predict AECOPD compared to using either one of them alone. A possible explanation could be that the two biomarkers represent different COPD phenotypes with different pathways leading to an exacerbation. One such phenotype could be concurrent chronic bronchitis, in which B-Eos but not NLR could prognosticate AECOPD. However, these findings must be interpreted cautiously, as our cohort probably was too small to divide into even smaller strata without losing power and the comorbidities were self-reported.

A biomarker used for long-term prediction in a stable phase of disease should be reliable and repeatable over time [12]. Regarding NLR, $10.6 \%$ of our study population had persistently high values. The ICC was 


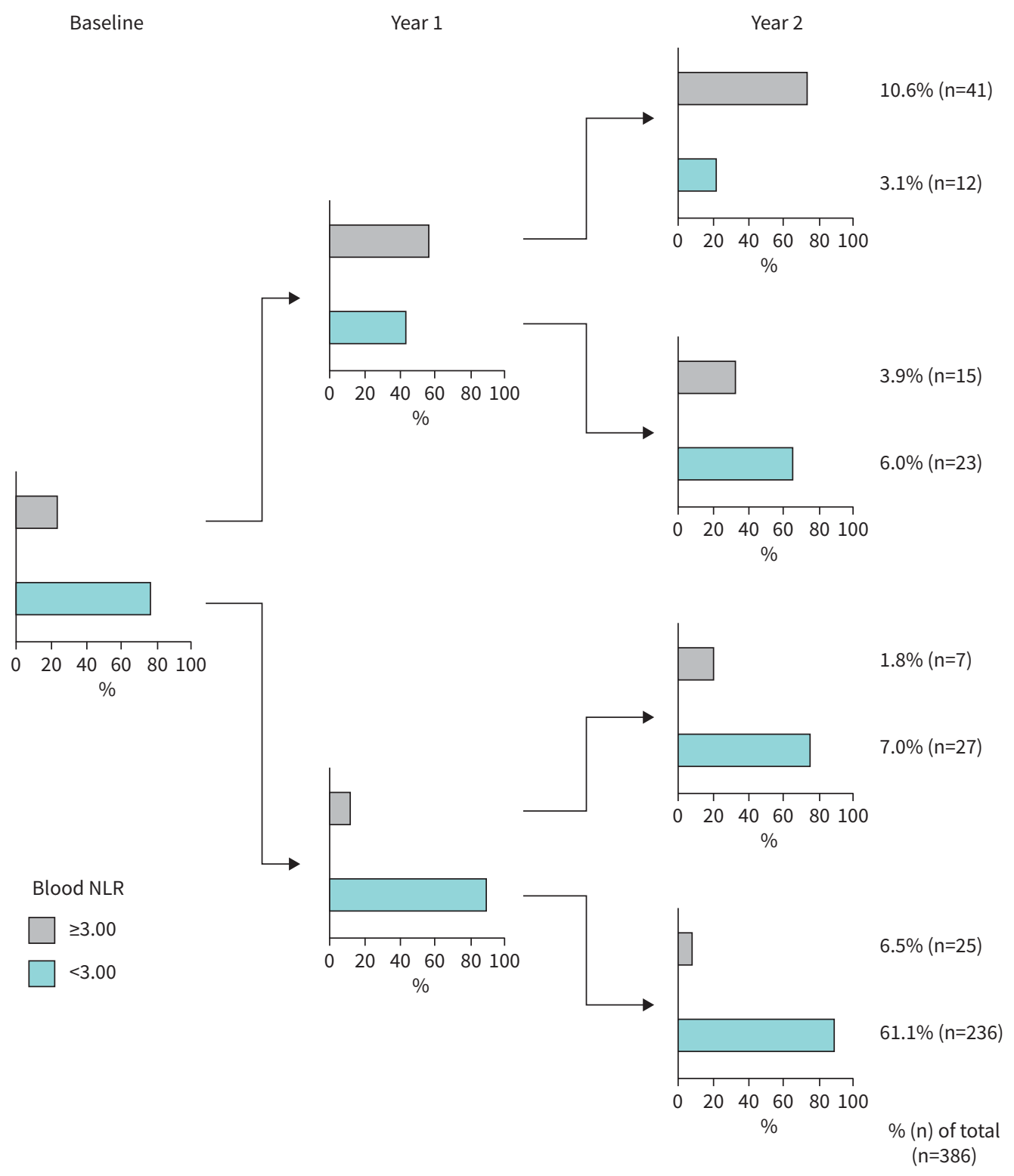

FIGURE 3 Longitudinal stability of blood neutrophil-to-lymphocyte ratio (NLR) in 386 subjects with COPD. The diagram at the left shows the proportion of subjects with high and low neutrophil-to-lymphocyte ratio at baseline visit. The diagrams in the middle show the respective proportion at year 1 , and the diagrams at the right show the respective proportion at year 2 .

0.61, indicating fair reliability. To our knowledge, GreEn et al.'s [39] study is the only prior study that has used ICC to assess the reliability of repeated NLR-measurements. In a retrospective cohort of 558 patients with two blood samples drawn 8-99 days apart before cardiac surgery, they found an ICC remarkably close to ours at 0.59 [39]. However, their cohort contained only 15 patients (2.7\%) with unspecified chronic lung disease. Regardless, our finding of a similar ICC in a different cohort with a longer follow-up time adds to the limited knowledge of NLR's temporal reliability. These results indicate that a single NLR value is quite representable for an individual with stable-phase COPD, and that NLR has potential as a biomarker in the sense that it is fairly repeatable.

Regarding B-Eos, 15.3\% of our study population had persistently high stable-phase values. This is in line with most previous works reporting that about $12-20 \%$ of COPD subjects have persistently high B-Eos during 1-3 years [8, 23-25]. Two studies differ with 5\% [22] and 45\% [21] of subjects with persistently 


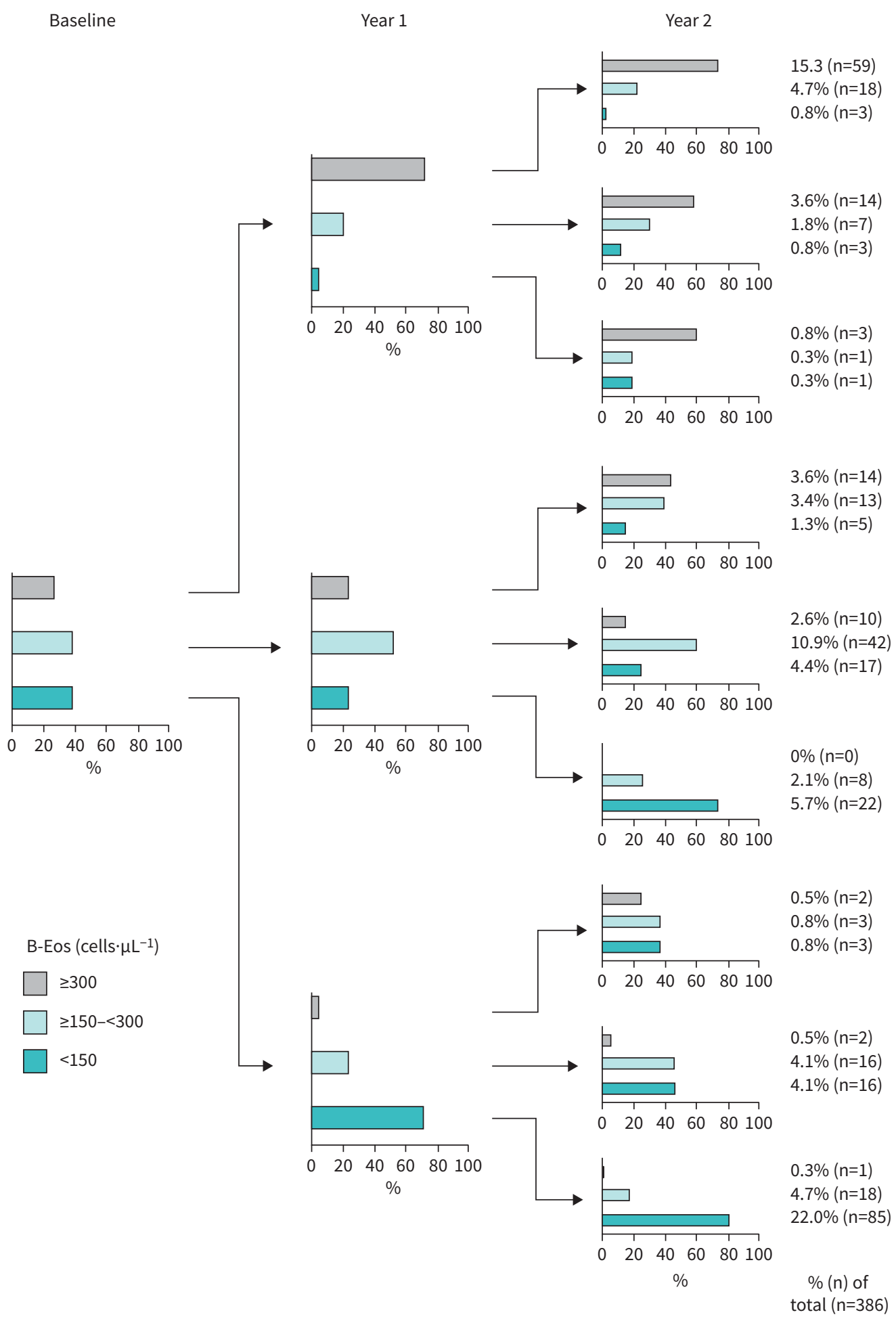

FIGURE 4 Longitudinal stability of blood eosinophil levels in 386 subjects with COPD. The diagram at the left shows the proportion of subjects with high, intermediate and low blood eosinophils (B-Eos) at baseline visit. The diagrams in the middle show the respective proportion at year 1 , and the diagrams at the right show the respective proportion at year 2 .

high B-Eos over 1.5-2 years. The latter study, however, considered AECOPDs only at baseline (i.e. during follow-up, blood samples drawn during an AECOPD might have been included), whereas all other studies included stable-phase measurements only. 

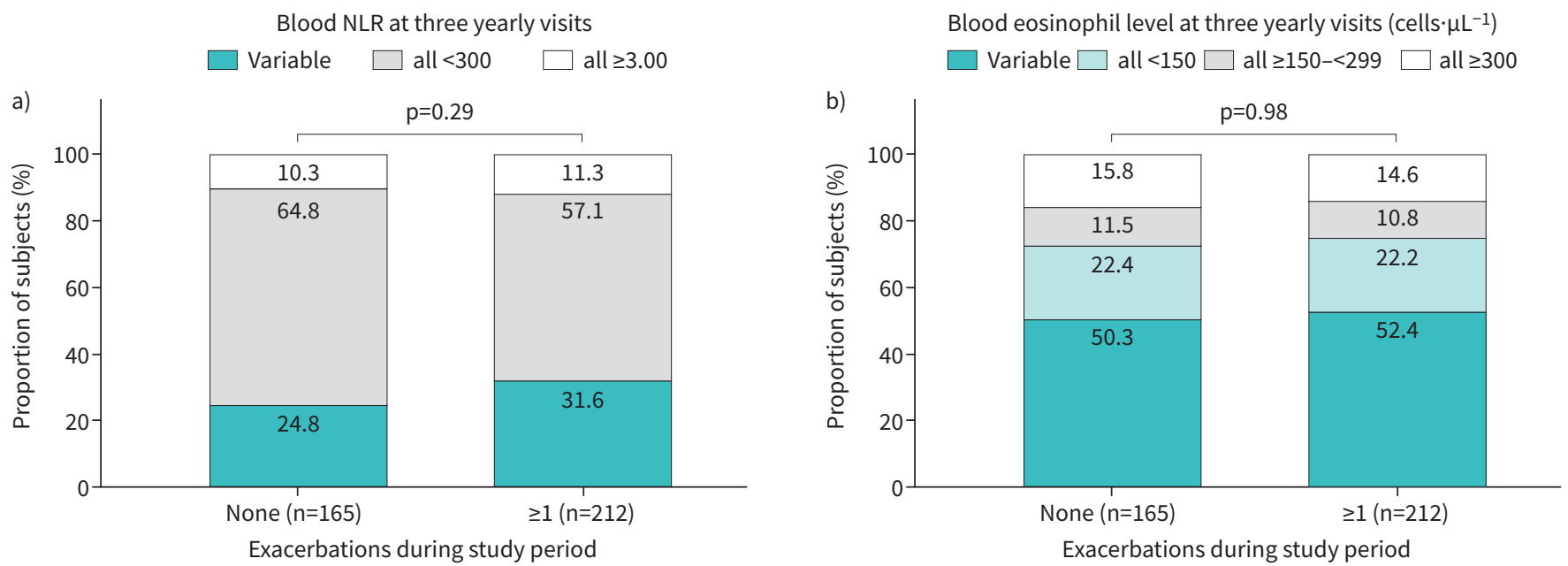

FIGURE 5 Longitudinal stability of a) blood neutrophil-to-lymphocyte ratio (NLR) and b) blood eosinophils measured at three yearly visits in 377 subjects with COPD, stratified by the occurrence of any acute exacerbation of COPD during the 3-year study period.

In our study, the ICC for B-Eos measurements was 0.69 , indicating good reliability. This is in line with previous papers reporting ICCs of 0.74-0.87 [25-27]. However, comparisons between our ICC and the previously reported ones are difficult, since none of the latter specifies which type of ICC has been calculated [33], and none present confidence intervals. Nonetheless, they all point in the same direction and suggest that the longitudinal reliability of B-Eos in stable-phase COPD is at least fair.

The major strengths of this study were the thorough follow-up of subjects and the relatively low dropout rate. Moreover, the examination of all subjects with spirometry ensured that the COPD diagnosis was correct. By including subjects from both primary and secondary care and several Swedish regions, the chance of a cohort representative for Swedish COPD patients increased and thus also the likelihood that our results are generalisable.

A major limitation was that our outcome (AECOPD) was self-reported only, which confers doubt on the accuracy. Moreover, the questionnaires used precluded counting of the number of AECOPDs, forcing us to treat it as a dichotomous variable and thus not allowing us to account for the frequency of AECOPDs in our analyses. The date of AECOPD was also lacking in the questionnaires, precluding any time-dependent analyses. In addition, there were no data on AECOPDs after year 2, which hindered prospective examination of associations between blood cell stability groups and outcomes such as AECOPD. Further limitations were that available data on comorbidities were self-reported, and that comorbidities such as bronchiectasis and gastro-oesophageal reflux were not included in the questionnaires. Moreover, no information on respiratory tract infections during the study period was recorded. Furthermore, subjects included in our analyses generally had less severe COPD and better health status than those not included. Only about $5 \%$ of the study population had very severe COPD, which raises concern about the generalisability of our findings to this severity group. Moreover, the precision in reporting of B-Eos results differed between study sites, resulting in an over-representation of exact "hundreds" of cells $\mu \mathrm{L}^{-1}$. Finally, using different laboratory equipment and routines also confer a certain risk of bias, although all methods employed were clinically approved and in routine clinical use. However, clustering of subjects based on study sites did not significantly change estimates of the mixed-effects regression models, indicating that any differences between study sites were of no relevance.

In conclusion, there was a clear albeit weak association between stable-phase NLR and future AECOPD in this study, especially in subjects with a history of AECOPD. High stable-phase B-Eos was associated with future AECOPD. Stable-phase NLR on its own is probably not useful to predict AECOPDs but may play a role as part of a risk scoring index. More work is needed to elucidate the clinical usefulness of NLR in stable-phase COPD and to establish relevant cut-off values. A minority of subjects with COPD had persistently elevated stable-phase NLR or B-Eos, but the biomarkers showed fair to good longitudinal reliability, which is desirable for factors informing clinical decisions. 
Acknowledgements: The authors would like to thank the personnel at the study sites without whom this work would have been impossible.

Provenance: Submitted article, peer reviewed.

Data sharing statement: Due to Swedish personal data protection legislation, study data cannot be freely shared. After ethical approval, anonymised patient-level data will be made available by the corresponding author.

Author contributions: C. Janson, K. Bröms, K. Lisspers, B. Ställberg, M. Högman and A. Malinovschi initiated and designed the TIE-study. J. Ellingsen, $C$. Janson and A. Malinovschi conceived the present study. Data collection: C. Janson, K. Bröms, K. Lisspers, B. Ställberg, M. Högman and A. Malinovschi. Statistical analysis: J. Ellingsen. Interpretation of data: all authors. Drafting and finalising the manuscript: J. Ellingsen. Critical revision of manuscript for intellectual content: all authors. All authors approve to the final version of the manuscript and agree to be accountable for all aspects of the work.

Conflict of interest: J. Ellingsen has received honoraria for lectures from AstraZeneca, Novartis and Teva; has served on advisory boards arranged by Novartis and GlaxoSmithKline and has received grants from Uppsala County Association against Heart and Lung Diseases and the Bror Hjerpstedt foundation. C. Janson reports no conflict of interest with regard to the present study. K. Bröms reports no conflict of interest with regard to the present study. K. Lisspers has received honoraria for lectures from AstraZeneca, Novartis, Boehringer Ingelheim and TEVA and served on advisory boards arranged by AstraZeneca, GlaxoSmithKline and Boehringer Ingelheim. B. Ställberg has received honoraria for educational activities and lectures from AstraZeneca, Boehringer Ingelheim, Meda/Mylan, Novartis, Chiesi and Teva, and has served on advisory boards arranged by AstraZeneca, Novartis, Meda, GlaxoSmithKline, Teva, and Boehringer Ingelheim, M. Högman reports no conflict of interest with regard to the present study. A. Malinovschi reports no conflict of interest with regard to the present study.

Support statement: This study was funded by the Uppsala-Örebro Regional Research Council; Centre for Research and Development, Uppsala University/Region Gävleborg; Centre for Clinical Research, Uppsala University; County Council Dalarna; The Swedish Heart-Lung Foundation; The Swedish Heart and Lung Association; The Uppsala County Association against Heart and Lung Diseases; and the Bror Hjerpstedt Foundation. Funding information for this article has been deposited with the Crossref Funder Registry.

\section{References}

1 Roth GA, Abate D, Abate $\mathrm{KH}$, et al. Global, regional, and national age-sex-specific mortality for 282 causes of death in 195 countries and territories, 1980-2017: a systematic analysis for the Global Burden of Disease Study 2017. Lancet 2018; 392: 1736-1788.

2 Global Initiative for Chronic Obstructive Lung Disease (GOLD). Global Strategy for the Diagnosis, Management and Prevention of Chronic Obstructive Pulmonary Disease Report. 2021. https://goldcopd.org/ 2021-gold-reports/ Date last accessed: 3 March 2021. Date last updated: 25 November 2020.

3 Aaron SD, Vandemheen KL, Clinch JJ, et al. Measurement of short-term changes in dyspnea and disease-specific quality of life following an acute COPD exacerbation. Chest 2002; 121: 688-696.

4 Soler-Cataluna JJ, Martinez-Garcia MA, Roman Sanchez P, et al. Severe acute exacerbations and mortality in patients with chronic obstructive pulmonary disease. Thorax 2005; 60: 925-931.

5 Larsson $\mathrm{K}$, Janson $\mathrm{C}$, Lisspers $\mathrm{K}$, et al. The impact of exacerbation frequency on clinical and economic outcomes in Swedish COPD patients: the ARCTIC study. Int J Chron Obstruct Pulmon Dis 2021; 16: 701-713.

6 Vedel-Krogh S, Nielsen SF, Lange P, et al. Blood eosinophils and exacerbations in chronic obstructive pulmonary disease. The Copenhagen general population study. Am J Respir Crit Care Med 2016; 193: 965-974.

7 Oshagbemi OA, Franssen FME, Braeken DCW, et al. Blood eosinophilia, use of inhaled corticosteroids, and risk of COPD exacerbations and mortality. Pharmacoepidemiol Drug Saf 2018; 27: 1191-1199.

8 Casanova C, Celli BR, de-Torres JP, et al. Prevalence of persistent blood eosinophilia: relation to outcomes in patients with COPD. Eur Respir J 2017; 50: 1701162

9 Bafadhel M, Peterson S, De Blas MA, et al. Predictors of exacerbation risk and response to budesonide in patients with chronic obstructive pulmonary disease: a post-hoc analysis of three randomised trials. Lancet Respir Med 2018; 6: 117-126.

10 Pascoe S, Barnes N, Brusselle G, et al. Blood eosinophils and treatment response with triple and dual combination therapy in chronic obstructive pulmonary disease: analysis of the IMPACT trial. Lancet Respir Med 2019; 7: 745-756.

11 Barnes PJ. Inflammatory endotypes in COPD. Allergy 2019; 74: 1249-1256.

12 Tine $M$, Biondini $D$, Semenzato $U$, et al. Reassessing the role of eosinophils as a biomarker in chronic obstructive pulmonary disease. J Clin Med 2019; 8: 962. 
13 Barnes NC, Sharma R, Lettis S, et al. Blood eosinophils as a marker of response to inhaled corticosteroids in COPD. Eur Respir J 2016; 47: 1374-1382.

14 Pavord ID, Chanez P, Criner GJ, et al. Mepolizumab for eosinophilic chronic obstructive pulmonary disease. N Engl J Med 2017; 377: 1613-1629.

15 Criner GJ, Celli BR, Brightling CE, et al. Benralizumab for the prevention of COPD exacerbations. N Engl J Med 2019; 381: 1023-1034.

16 Vaguliene N, Zemaitis M, Lavinskiene S, et al. Local and systemic neutrophilic inflammation in patients with lung cancer and chronic obstructive pulmonary disease. BMC Immunol 2013; 14: 36.

17 Gunay E, Sarinc Ulasli S, Akar O, et al. Neutrophil-to-lymphocyte ratio in chronic obstructive pulmonary disease: a retrospective study. Inflammation 2014; 37: 374-380.

18 Paliogiannis P, Fois AG, Sotgia S, et al. The neutrophil-to-lymphocyte ratio as a marker of chronic obstructive pulmonary disease and its exacerbations: a systematic review and meta-analysis. Eur J Clin Invest 2018; 48: e12984.

19 Pascual-González Y, López-Sánchez M, Dorca J, et al. Defining the role of neutrophil-to-lymphocyte ratio in COPD: a systematic literature review. Int J Chron Obstruct Pulmon Dis 2018; 13: 3651-3662.

20 Ye Z, Ai X, Liao Z, et al. The prognostic values of neutrophil to lymphocyte ratio for outcomes in chronic obstructive pulmonary disease. Medicine 2019; 98: e16371.

21 Oshagbemi OA, Burden AM, Braeken DCW, et al. Stability of blood eosinophils in patients with chronic obstructive pulmonary disease and in control subjects, and the impact of sex, age, smoking, and baseline counts. Am J Respir Crit Care Med 2017; 195: 1402-1404.

22 Greulich T, Mager S, Lucke T, et al. Longitudinal stability of blood eosinophil count strata in the COPD COSYCONET cohort. Int J Chron Obstruct Pulmon Dis 2018; 13: 2999-3002.

23 Landis S, Suruki R, Maskell J, et al. Demographic and clinical characteristics of COPD patients at different blood eosinophil levels in the UK clinical practice research datalink. COPD 2018; 15: 177-184.

24 Shin SH, Park HY, Kang D, et al. Serial blood eosinophils and clinical outcome in patients with chronic obstructive pulmonary disease. Respir Res 2018; 19: 134.

25 Southworth T, Beech G, Foden P, et al. The reproducibility of COPD blood eosinophil counts. Eur Respir J 2018; 52: 1800427.

26 Landis SH, Suruki R, Hilton E, et al. Stability of blood eosinophil count in patients with COPD in the UK clinical practice research datalink. COPD 2017; 14: 382-388.

27 Long GH, Southworth T, Kolsum U, et al. The stability of blood Eosinophils in chronic obstructive pulmonary disease. Respir Res 2020; 21: 15.

28 Sakurai $\mathrm{K}$, Chubachi $\mathrm{S}$, Irie $\mathrm{H}$, et al. Clinical utility of blood neutrophil-lymphocyte ratio in Japanese COPD patients. BMC Pulm Med 2018; 18: 65.

29 Hogman M, Sulku J, Stallberg B, et al. 2017 Global initiative for chronic obstructive lung disease reclassifies half of COPD subjects to lower risk group. Int J Chron Obstruct Pulmon Dis 2018; 13: 165-173.

30 Jones PW, Harding G, Berry P, et al. Development and first validation of the COPD assessment test. Eur Respir J 2009; 34: 648-654.

31 Hedenström H, Malmberg P, Agarwal K. Reference values for lung function tests in females. Regression equations with smoking variables. Bull Eur Physiopathol Respir 1985; 21: 551-557.

32 Hedenström $\mathrm{H}$, Malmberg P, Fridriksson HV. Reference values for lung function tests in men: regression equations with smoking variables. Ups J Med Sci 1986; 91: 299-310.

33 Koo TK, Li MY. A guideline of selecting and reporting intraclass correlation coefficients for reliability research. J Chiropr Med 2016; 15: 155-163.

34 Lee $\mathrm{H}, \mathrm{Um} \mathrm{SJ}$, Kim YS, et al. Association of the neutrophil-to-lymphocyte ratio with lung function and exacerbations in patients with chronic obstructive pulmonary disease. PLOS ONE 2016; 11: e0156511.

35 Lee SJ, Lee HR, Lee TW, et al. Usefulness of neutrophil to lymphocyte ratio in patients with chronic obstructive pulmonary disease: a prospective observational study. Korean J Intern Med 2016; 31: 891-898.

36 Azab B, Camacho-Rivera M, Taioli E. Average values and racial differences of neutrophil lymphocyte ratio among a nationally representative sample of United States subjects. PLOS ONE 2014; 9: e112361.

37 Turato G, Semenzato U, Bazzan E, et al. Blood eosinophilia neither reflects tissue eosinophils nor worsens clinical outcomes in chronic obstructive pulmonary disease. Am J Respir Crit Care Med 2018; 197: 1216-1219.

38 Singh D. Blood eosinophil counts in chronic obstructive pulmonary disease: a biomarker of inhaled corticosteroid effects. Tuberc Respir Dis 2020; 83: 185-194.

39 Green J, Bin Mahmood SU, Mori M, et al. Stability across time of the neutrophil-lymphocyte and lymphocyte-neutrophil ratios and associations with outcomes in cardiac surgery patients. $J$ Cardiothorac Surg 2019; 14: 164. 